$\Delta$ Palabras clave/ Innovación, educación, proyecto, holística.

$\Delta$ Keywords/ Innovation, education, project, holistic.

$\Delta$ Recepción/ 3 abril 2018

$\Delta$ Aceptación/ 10 julio 2018

\section{Taller de sólidos platónicos. Transferencia del modelo del proyecto en arquitectura a la Enseñanza Media.}

\section{Platonic solids workshop. Taking the project model from architecture to high-school.}

\section{Rodrigo Lagos-Vergara}

Arquitecto, Universidad del Bío-Bío, Chile.

Master "Arte, Arquitectura y Ciudad", Universidad Politécnica de Cataluña, España.

Académico, Departamento de Diseño y Teoría de la Arquitectura, Universidad del Bío-Bío, Chile rlagos@ubiobio.cl

\section{Magaly Mella-Abalos}

Antropóloga, Universidad Academia de

Humanismo Cristiano, Chile.

Diploma de Estuudios Avanzados en

Antropología Social y Cultural, Universitat de

Barcelona, España

Profesional Investigadora, Centro de Estudios

Urbano Regionales, Universidad del Bío-Bío,

Chile.

magmella@ubiobio.cl

\section{María Verónica-Strocchi}

Licenciada en Letras Modernas, Universidad Nacional de Córdoba, Argentina.

Licenciada en Lengua y Literatura Hispánica

Universidad de Chile, Chile.

Profesora de Lenguaje y Comunicación

Universidad del Desarrollo, Chile.

mvstrocchiaudd.cl

RESUMEN/ El modelo educativo de la Enseñanza Media en Chile se basa en prácticas y estrategias anacrónicas que no logran materializar en el aula la declarada educación integral, lo que afecta el aprendizaje y la emocionalidad de los estudiantes. El objeto de estudio de esta investigación es auscultar el impacto de una propuesta didáctica innovadora: el Taller de Sólidos Platónicos, que supone la transferencia a la educación media de una metodología característica de la arquitectura -el aprendizaje basado en proyecto-, conjugado con un enfoque holístico de la educación que busca el desarrollo de las diferentes áreas del individuo y un abordaje complejo y no segmentado del conocimiento. Para el desarrollo de una propuesta de formación integral u holística, adquieren importancia las bio-herramientas, entendidas como herramientas de enseñanza y terapia que implican la integración de los diferentes planos que componen el ser humano, tales como el físico, mental, emocional y espiritual. ABSTRACT/ The Chilean high-school education model is based on outdated practices and strategies unable to bring to classrooms what has been called holistic education, with the ensuring impact on students' learning and emotions. This research is aimed at discussing the impact of an innovative teaching practice: the Platonic Solids Workshop, which takes to the high-school level a methodology typical of architecture -project-based learning- coupled with a holistic approach to education that aims at developing the different realms of an individual and a complex and non-segmented approach to knowledge. Relevant to the development of this holistic training proposal are bio-tools, understood as teaching and therapy tools that involve integrating the different aspects of being human: physical, mental, emotional and spiritual.

\section{INTRODUCCIÓN}

A partir de 1989, Chile inició un proceso de cambio y reformas en materia de educación que, en términos generales, se podrían circunscribir a dos períodos. El primero abarca desde 1990 hasta el año 2005, cuando los principios fundamentales se orientaron a incidir en la eficiencia, calidad y equidad de la educación (Román 2003), atendiendo casi exclusivamente a las deficiencias del sistema en sus aspectos económicos y materiales (Brovetto

1999). El segundo período, que prevalece - hasta nuestros días, se inicia el año 2006 con el cuestionamiento a la calidad de la enseñanza, así como también a la inequidad y exclusión que caracterizaban a la educación chilena (Galdames, Moffat y Cisternas 2014). Este movimiento, que encabezaron los estudiantes secundarios, fue respaldado en los años siguientes por los universitarios (2011) y los docentes (2014). Lo anterior ha repercutido en el inicio de la discusión de un conjunto de reformas que, hasta la fecha, han buscado generar una transformación social en todos los niveles del sistema de educación y territorios del país.
El año 2013, la Universidad del Bío-Bío inició el convenio de desempeño de Mejoramiento de la Calidad de la Educación Superior del Ministerio de Educación (MECESUP), Sistema Territorial de Educación. Dicho programa se propone implementar escalonadamente un sistema de educación territorial, de bien público, que aporte a la formación de capital humano y social. Uno de sus componentes es el Observatorio Prospectivo de la Educación y el Desarrollo Regional, espacio de análisis y reflexión participativa sobre los nuevos caminos que debe enfrentar la educación regional y el 
desarrollo del territorio, indagando futuros posibles y deseables (Galdames et al. 2014) y discutiendo sobre diferentes temáticas, entre las que destaca la necesidad de implementar prácticas pedagógicas innovadoras sustentadas en los nuevos paradigmas educacionales (Galdames et al. 2014).

En un contexto educativo donde existe un currículum escolar segmentado, centrado en unas pocas habilidades cognitivas que margina a quienes son más fuertes en otras inteligencias, el aprendizaje a través del proyecto -en las artes y la arquitecturaofrece más opciones para una educación integral, al promover el desarrollo de un conjunto de habilidades que el sistema dominante no contempla. Sin embargo, las condiciones para el desarrollo de estas son muy deficientes, particularmente en la educación pública (Rojas 2017), perpetuándose su marginalidad y fragilidad dentro del sistema escolar. Así, existen más formas de representación que utilizan una sintaxis más bien figurativa (metáforas) en lugar de aquellas gobernadas por las reglas (códigos). De acuerdo a Eisner, "las reglas para decodificar los códigos son específicas mientras que para explicar las metáforas se requiere de imaginación" (1994: 89). Para Rojas (2017), el modelo dominante de la didáctica escolar es el de códigos y no el de producción no reglada de metáforas. Este último es el campo de las artes y de la representación proyectual.

Este artículo se refiere a una experiencia exploratoria de innovación en educación que, desde una mirada interdisciplinaria, busca abrir caminos sobre nuevas metodologías de enseñanza. La investigación se orienta a conocer cómo el modelo didáctico de taller proyectual -una experiencia de innovación denominada "Sólidos Platónicos", que se realiza cada año, desde 2012, a estudiantes de primer ciclo de la carrera de Arquitectura de la Universidad del Bío-Bío-, explorando un nuevo abordaje integrador y transdisciplinar e introduciendo elementos del enfoque holístico en educación, es valorado por estudiantes y por docentes de la enseñanza media. Para lo anterior, se optó por reproducir el modelo con un grupo de estudiantes de tercero y cuarto medio del Liceo Bicentenario Isidora Ramos (Lebu, Provincia de Arauco, Región del Biobío, Chile) y, posteriormente, replicar la acción con un grupo de docentes del mismo establecimiento. Para lo anterior nos planteamos la siguiente pregunta: ¿Qué resultados genera la transferencia de una experiencia de innovación pedagógica con enfoque holístico, de la Universidad del Bío-Bío (taller de proyectos de arquitectura "Sólidos Platónicos"), en estudiantes y

\section{docentes de este liceo?}

\section{MARCO TEÓRICO: Paradigma de la complejidad y su vinculación con el enfoque holístico e integral de la educación. La orientación de} esta investigación aplicada se adscribe al paradigma crítico, donde la reflexión teórica adquiere fuerza transformadora, al interactuar permanentemente con las acciones ejecutadas, partiendo del supuesto de que las diferentes esferas del hacer humano están sujetas al cambio y que el proceso de investigación ofrece posibilidades de mejorar la realidad estudiada. Barfield (2001) sostiene que los modelos educativos varían culturalmente, siendo la escolarización una forma adoptada en la época moderna y en el contexto occidental. La educación constituye, por lo tanto, una institución universal e histórica, que es permeable a los cambios del entorno, donde -como diría Deweyeducar basándose en circunstancias pasadas es como adaptar un organismo a un ambiente que ya no existe (Dewey 1965). En este sentido, se puede afirmar que el actual sistema educativo, cuyos fundamentos se vinculan con la visión de mundo de la sociedad decimonónica de mirada positivista, no logra dar respuesta a necesidades de la sociedad contemporánea (Lagos 2013, 2012).

La actual etapa histórica ha sido denominada sociedad en red o sociedad de la información, pues una de sus características esenciales es la posibilidad de obtener información y de acceder a diversos contenidos de manera instantánea, desde cualquier lugar y con un costo muy bajo. Esto implica que los estudiantes, durante su proceso educativo, pueden acceder a los diversos ambientes de aprendizaje provistos por las tecnologías. En este escenario, el aprendizaje se vuelve ubicuo y la institución educativa deja de ser el espacio exclusivo de difusión del saber, por lo que su función no puede limitarse a la trasmisión de contenidos exclusivamente conceptuales y a la formación de

corte cognitivo

El relato moderno, caracterizado por su desvinculación con los procesos culturales y en su forma de concebir a la persona, fundamentó el modelo educativo tradicional (Morin 1999). La emergencia del paradigma de la complejidad (Morin 1994) cuestiona la efectividad e impacta la práctica pedagógica, modificando el concepto de conocimiento. A grandes rasgos, busca articular e integrar el conocimiento desde diferentes áreas, tales como la científica, humanista y artística, así como vincular el concepto de paradigma de la complejidad con el de perspectiva holística. En este sentido, resulta clave la noción de circuito epistemológico, que plantea la necesaria integración del conocimiento mediante la adopción de una perspectiva trans e interdisciplinaria, propiciando un abordaje complejo y no segmentado del conocimiento, generando un marco conceptual que permita establecer interrelaciones e intercomunicaciones reales entre las diversas disciplinas, y provocando un diálogo fecundo entre especialistas, metodologías y lenguajes específicos (Barberousse 2008). 
Desde el punto de vista educativo, la formación integral supone que toda persona encuentra su identidad, significado y sentido de vida a través de nexos con la comunidad, el mundo natural y el desarrollo de valores humanos. La educación es el proceso de aprendizaje permanente que abarca las distintas etapas de la vida de las personas. Tal como entenderemos la perspectiva holística, que se aparta profundamente de la visión positivista:

"...una educación o un sistema de crecimiento personal y grupal que implique, entienda y atienda de manera armónica e integrada los diferentes ámbitos: el desarrollo físico emocional, cognitivo, ecológico, estético-creador, intuitivo, espiritual, social, multicultural, y éticosolidario" (Paymal 2012: 7)

Una comprensión holística de la realidad implica una voluntad de comprensión global que tiende a vincular las diferentes áreas o disciplinas, intentando favorecer un análisis estructural no fragmentario y una relación maximizada entre el todo y sus partes (Santos 2000). Desde esta perspectiva, es interesante constatar el surgimiento de lo que Paymal ha llamado "bio-herramientas", y que hace referencia a herramientas de enseñanza y de terapia enfocadas, fundamentalmente, en el desarrollo integral del ser humano (Paymal 2010). Estos instrumentos se basan en el uso de técnicas de activación, tanto de los cinco sentidos exteriores como de las áreas creativas e intuitivas, vinculadas al hemisferio cerebral derecho. En la base de estas propuestas está la teoría del cerebro triuno o de los tres cerebros -neo Cortex, límbico y reptil- (Mac Lean 1990), relacionada con la coherencia en el pensar, sentir y actuar del ser humano como ser físico, mental, emocional y espiritual.
TALLER DE SÓLIDOS PLATÓNICOS: Una propuesta de instrumentalización de la perspectiva holística e integral

en educación. La formación en el campo

de la arquitectura posee una didáctica particular -la del proyecto- y una estrategia pedagógica. El taller es el centro de la formación y el lugar donde el estudiante integra diferentes materias y saberes, adquiriendo habilidades y competencias para la vida profesional, a través de lo que Donald Schön Ilama la "reflexión en la acción" (1992), esto es, la capacidad del propio sujeto de construir de manera activa conocimiento a partir del hacer. La formación del arquitecto a través del proyecto considera el desarrollo integral del estudiante para el logro de aprendizajes significativos y su capacidad de reconocer las relaciones que existen entre las diferentes materias y el mundo que les rodea, adaptándose a situaciones nuevas y combinando conocimientos para resolver problemas reales o simulados

La Escuela de Arquitectura de la Universidad del Bío-Bío ha elaborado las bases de un modelo didáctico, el taller de "Sólidos Platónicos", que se ha aplicado con muy buenos resultados desde 2012 (Lagos 2016). Este modelo hace del proyecto una experiencia significativa de reconexión con las formas primordiales de la naturaleza, que inicia a los estudiantes en el arte de la arquitectura. De acuerdo a los principios enunciados por Platón en el siglo V a.C., los sólidos platónicos son estructuras geométricas comunes a todas las cosas y las formas primordiales reveladas por la luz: así como las notas musicales son esenciales para el sonido, o los colores del espectro cromático para la luz, los sólidos son fundamentales para entender la forma en la naturaleza (Lagos 2016).

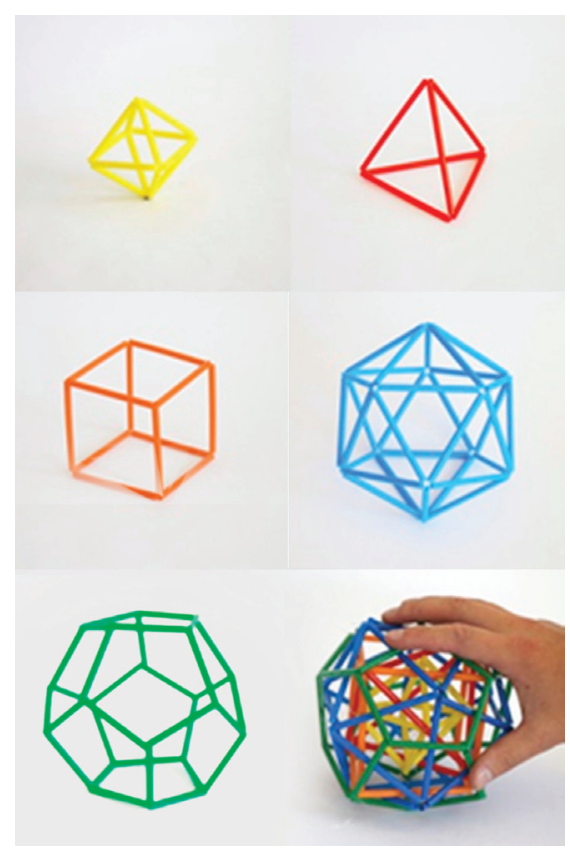

Imagen 1. Los 5 Sólidos Platónicos y la sobreposición de uno sobre otro sucesivamente (fuente: Archivos CEUR UBB).

El modelo didáctico de iniciación a la forma para el proyecto se enfoca en despertar en el estudiante tres nociones fundamentales: a) La conciencia holística de ser parte de un todo armónico dentro del mundo y el universo:

b) La comprensión de las leyes

universales de la naturaleza a través de la geometría tridimensional;

c) La distinción de los sólidos como las formas fundamentales de la naturaleza y como valores ancestrales constituyentes de las formas de la arquitectura y la ciudad (Lagos 2016). 


\section{METODOLOGÍA PROPUESTA: Recorrido de la investigación.}

La confiabilidad del estudio se basa en la utilización de métodos de investigación cualitativa y cuantitativa, circunscritas por la interdisciplinariedad de los investigadores y el empleo de técnicas de recolección de datos seleccionados en función del objetivo del estudio. Las técnicas empleadas fueron la observación etnográfica, la aplicación de una encuesta simple y el análisis de contenidos con la finalidad de triangular la información recabada y establecer hallazgos sobre la experiencia de innovación. Desde una mirada transdisciplinar, la investigación tiene tres momentos: 1. La implementación del taller de "Sólidos Platónicos".

2. La observación etnográfica del modelo de innovación.

3. La valoración del ejercicio que se registra en una encuesta simple y en el análisis del discurso.

La etnografía es un método que permite contar con un relato descriptivo a través la técnica de la observación participante (Mauss 2006; Hammersley y Atkinson 1994; Bengoa 1992; Geertz 1987). En este caso, se enfoca en un contexto educativo particular que se describe densamente. para luego interpretarlo (Geertz 1987), donde el autor pasa a ser un observador silencioso que deja hablar al testigo, objetivando lo subjetivo a través del texto, sin la pretensión de aprehender la realidad social sino que sugerirla reflexivamente (Bourdieu 2008) como un medio eficiente de obtención de conocimientos nuevos respecto de una situación social o cultural determinada (Hammersley y Atkinson 1994; Bengoa 1992). Posteriormente, se aplica una encuesta a los participantes del taller, estructurada mediante una secuencia de ítems cerrados que permiten establecer diferentes grados de valoración, a fin de conocer la satisfacción con la actividad. Dentro de esta se establece un apartado de análisis documental, para recopilar comentarios escritos de los participantes con el objetivo de generar categorías de análisis que derivan en los hallazgos.
PROGRAMA DEL TALLER "SÓLIDOS

PLATÓNICOS". El taller es dirigido por

un profesor y dos asistentes, quienes, al igual que los investigadores, permanecen durante todo el tiempo observando la actividad en los diferentes espacios en que se ejecuta. La duración aproximada es de dos horas y media, y para sistematizar la propuesta pedagógica se han diseñado dos secuencias, de manera de articular un programa que considera en su realización las diversas formas de aprendizaje de los estudiantes y la comprensión neurobiológica de las prácticas (Mac Clean 1990), hacia una integración y coherencia de los tres compuestos del cerebro.

\section{Primera parte: Experiencia de}

sensibilización y preparación para el aprendizaje desde el juego, que se realiza en los parques del campus universitario. Los participantes forman un círculo mirando hacia el centro, en rondas-espiral giratorias o tensadas, ejercitando simultáneamente el cerebro con la respiración y el enraizamiento. El profesor va guiando el trabajo, acorde a un relato que articula varios saberes del conocimiento, mediante bio-herramientas, articulando los cinco sentidos externos (vista, oído, olfato, gusto y tacto) y los cinco sentidos internos (el saber, el tiempo, la identidad, la conciencia multidimensional y el humor) (Paymal 2010). Los objetivos de estas prácticas son: desarrollar en los estudiantes capacidades relacionadas con el pensar, sentir y actuar coherente; desarrollar y ampliar en los estudiantes la percepción de sí mismos como seres físicos, mentales, emocionales y espirituales; y adquirir habilidades para una práctica diaria de encuentro con 'el aquí y el ahora'

Segunda parte: Trabajo de taller de "Sólidos Platónicos". El docente explica el objetivo de la actividad y de las acciones didácticas individuales, grupales y colectivas a seguir, que implican la construcción manual de los sólidos con varillas de globo y utilización de técnicas y bio-herramientas que despierten la curiosidad y motivación de los estudiantes, para potenciar habilidades tales como la creatividad, comunicación, pensamiento reflexivo y empatía (Lagos 2016).

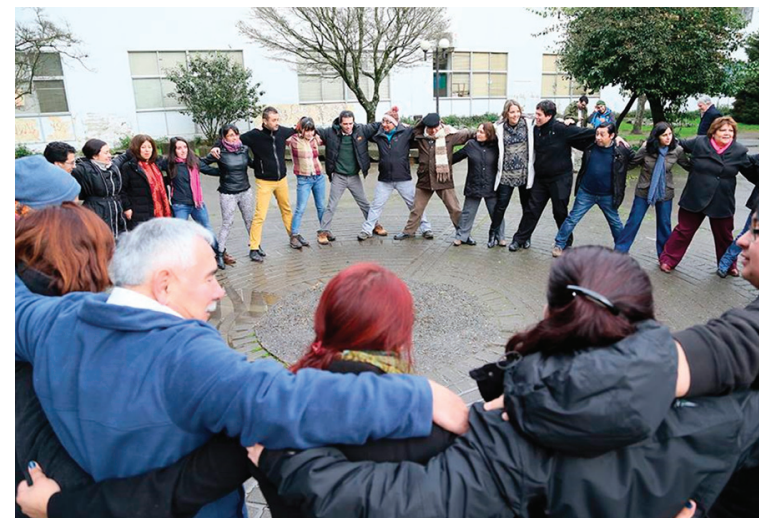

Imagen 2. Los docentes reaccionaron con espontaneidad durante el ejercicio al aire libre. Mentras hacian una ronda $y$ yeguian las instrucio a des del profesor gula, comenzaron a jug Archivos CEUR UBB).
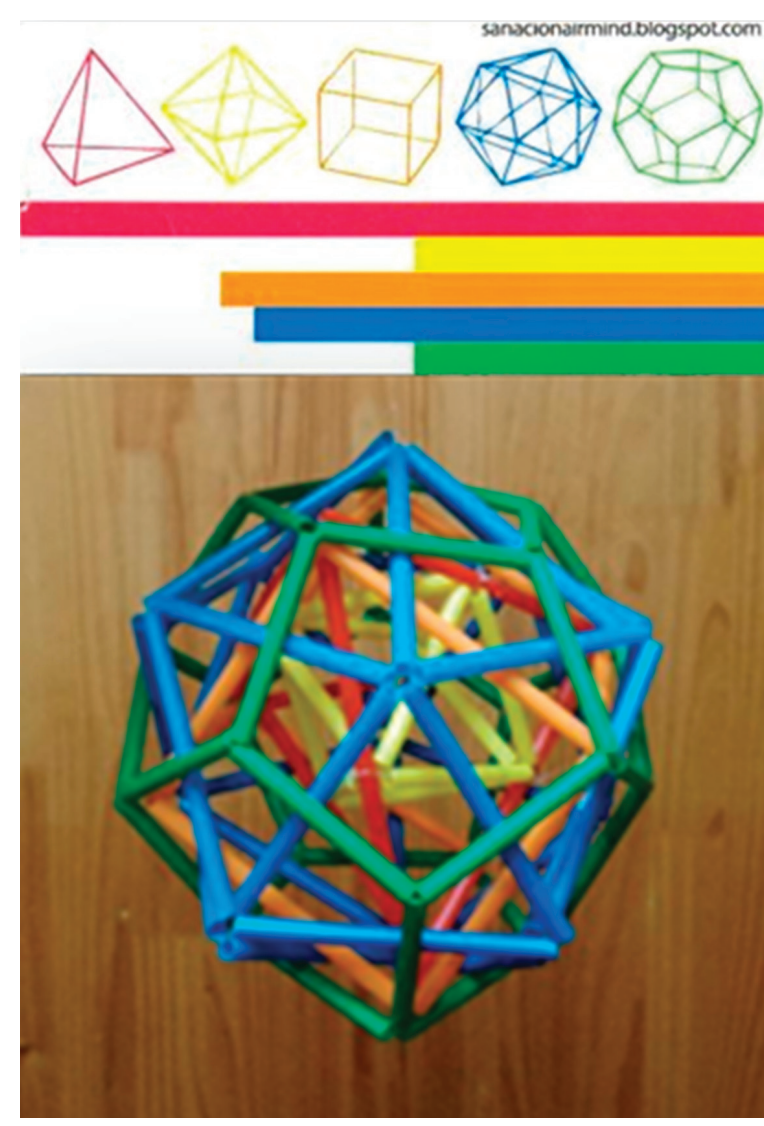

Imagen 3. Sólidos platónicos: arriba, plano para armar; abajo, model construido con la sobreposición de uno sobre otro en el orden anterior (fuente: Archivos CEUR UBB). 

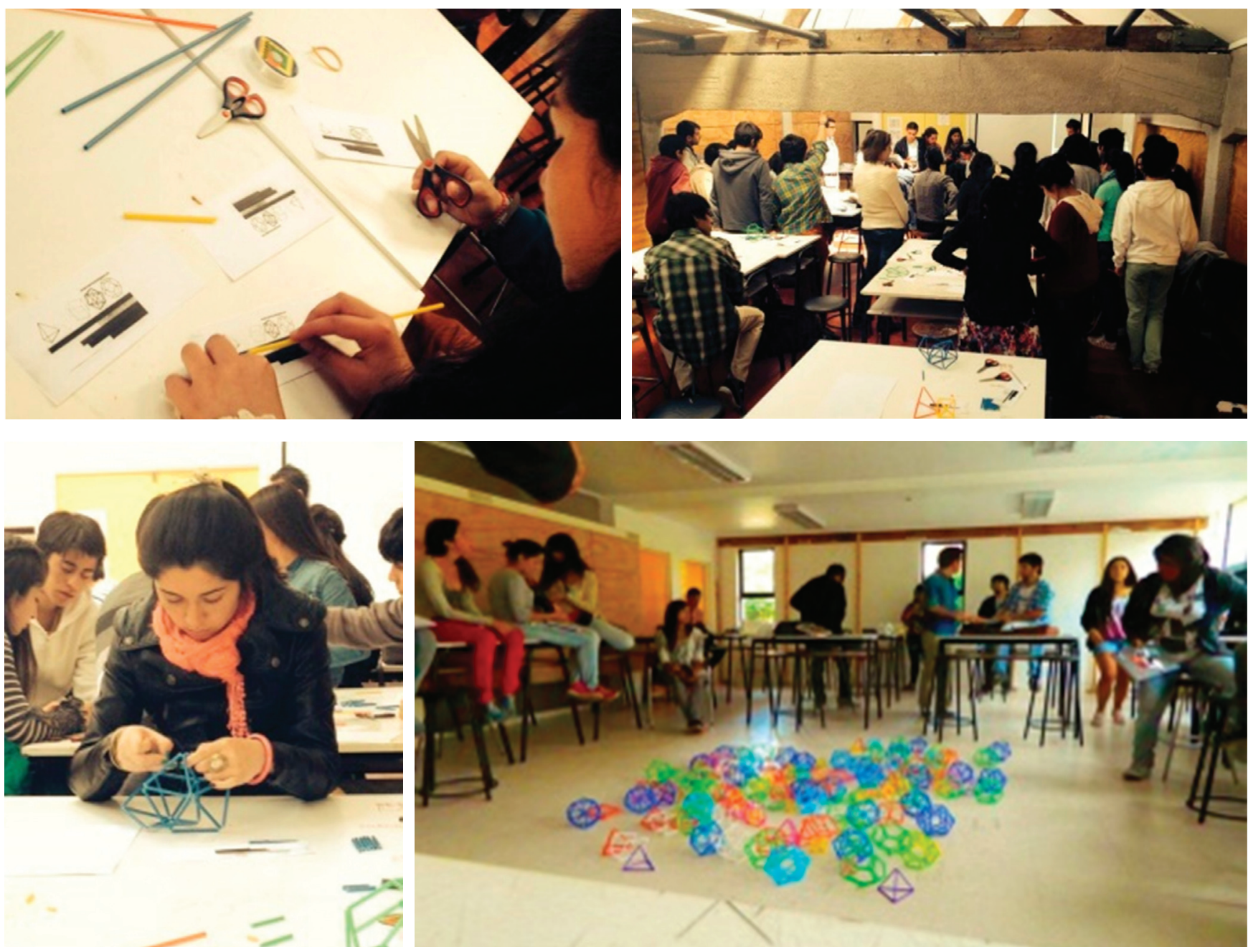

Imágenes 4 a 7. Construyendo sólidos en el taller (fuente: Archivos CEUR UBB).

\section{RESULTADOS DEL ANÁLISIS}

DOCUMENTAL. Luego de la aplicación

de la encuesta de satisfacción, el análisis

documental facilitó detectar valoraciones positivas y negativas, en relación a los ejes de temporalidad, motivación, experiencia de aprendizaje, proyección y docencia. Desde la visión de los estudiantes, hay una valoración negativa del tiempo destinado a la propuesta, basada en su insuficiencia para concluir con el proyecto del taller, esto es, el montaje superpuesto de las cinco formas básicas. La valoración positiva se vincula con la motivación, fundamentada en la apreciación de la propuesta como innovadora (diferente a lo conocido) y didáctica. El cambio se - instala en la forma de enseñanza, es decir ư en la propuesta metodológica que difiere de la clase expositiva tradicional; en este caso, se reemplaza por el empleo del aprendizaje basado en proyecto en el taller. La experiencia de aprendizaje no solo se identifica con la apropiación o desarrollo conceptual, sino que aparece vinculada al desarrollo de la creatividad e imaginación (habilidades cognitivas superiores).

En el caso de los docentes, la valoración negativa también se instala en el eje de la temporalidad. No obstante (y coincidente con su rol social), se elaboran propuestas de mejora, tales como aumentar los integrantes del grupo o modificar en el manejo del tiempo. La valoración positiva aparece vinculada a ejes de proyección, que suponen la replicabilidad del taller en otros contextos o con otras funciones. El eje de la emocionalidad se vincula con la inclusión de los sentimientos y/o estados anímicos, entendidos como factores relevantes en el proceso de enseñanza-aprendizaje. El eje de la proyección se relaciona con la posibilidad de adaptar la actividad a otros niveles de enseñanza.

En tanto experiencia de aprendizaje, el taller recibe una valoración positiva calificada como innovadora, tanto por la metodología utilizada (aprendizaje basado en proyecto, aprender haciendo, trabajo colaborativo) como por los resultados de aprendizaje obtenidos (desarrollo de habilidades y no solo de conceptos). Finalmente, se puede subrayar una alta valoración del desempeño docente, que destaca más el área afectiva y/o de competencias genéricas que el conocimiento propio de la disciplina. 

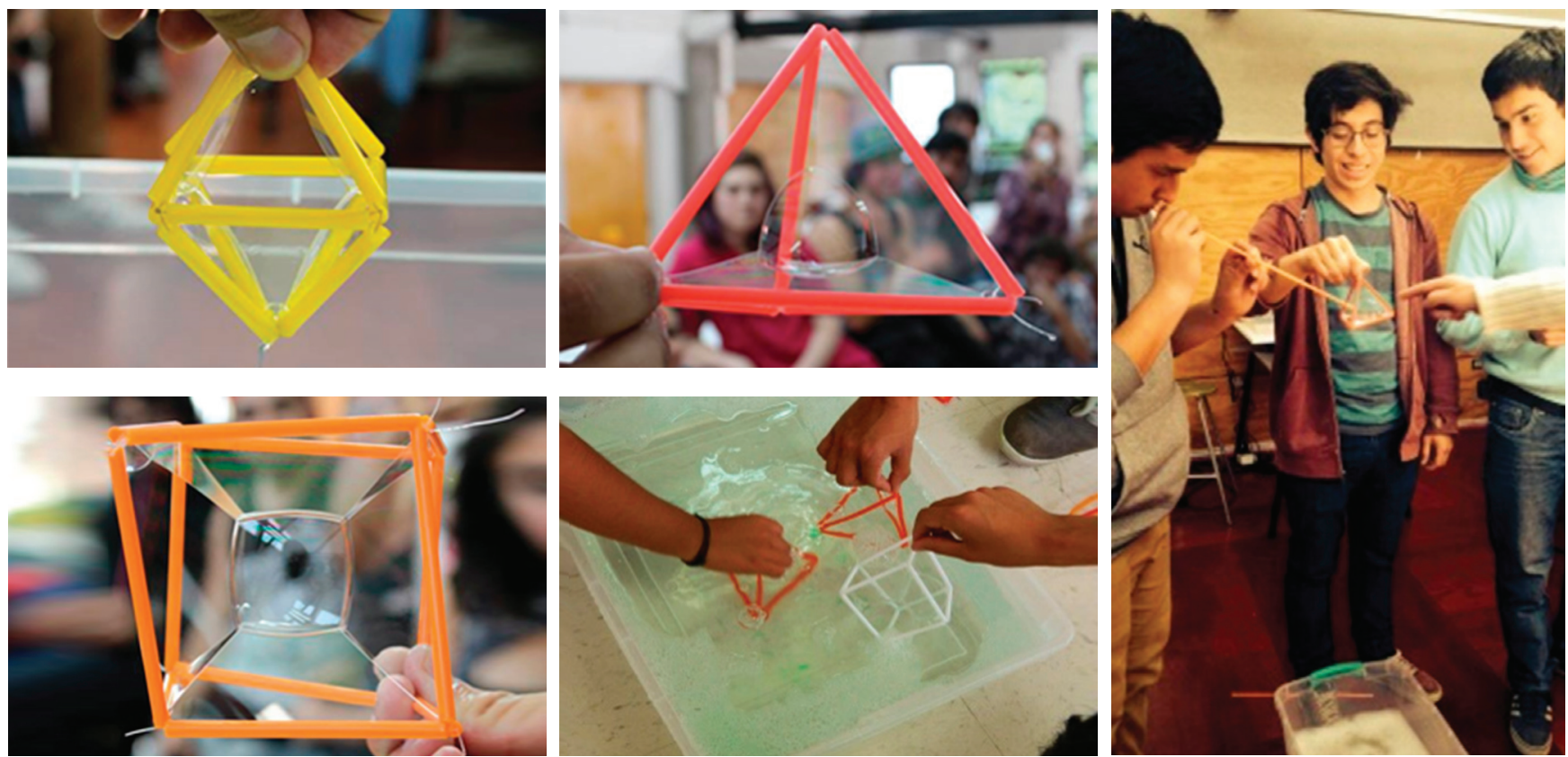

Imágenes 8 a 12. Los sólidos platónicos construidos con varillas se sumergen en solución de aqua con jabón haciendo aparecer, con las burbujas, las formas llamadas intrusiones (fuente: Archivos CEUR Imágen

\section{CONCLUSIONES, NUEVAS PREGUNTAS Y NUEVAS HIPÓTESIS.}

Tomando como base los resultados de

la investigación, y a partir del análisis realizado sobre los efectos y valoración del taller, tanto desde la descripción etnográfica -efectos observados desde el punto de vista metodológico, las similitudes y las diferencias detectables de la implementación del taller-, como desde la encuesta de valoración, se puede afirmar que la propuesta didáctica fue recibida satisfactoriamente tanto por estudiantes como por docentes. Los resultados de las encuestas indican una alta valoración, especialmente en los ítems referidos a motivación y desempeño docente. Tanto el relato etnográfico como el análisis del discurso, señalan que el taller constituye una innovación, en el sentido de que se aparta de las prácticas didácticas tradicionales. Para los estudiantes, la diferencia fundamental radica en el cambio de espacio (desplazamiento de la sala con pupitres individuales a un taller con mesas para trabajo grupal) y en el asombro como estrategia para motivar y, por ende, propiciar la construcción de conocimiento a partir de la motivación intrínseca.

Para los docentes, la innovación se vincula con la metodología utilizada, que supone una mirada transdisciplinar del conocimiento, que propicia la replicabilidad de la propuesta en las diferentes asignaturas establecidas por el currículum de la enseñanza media. También se destaca la inclusión de los estados anímicos como factor clave del aprendizaje, en esta experiencia propiciado de manera positiva por el empleo de bio-herramientas al inicio de la jornada. Se puede dar cuenta de otros resultados generados por la transferencia del taller, tales como:

1. La importancia del espacio y tiempo de ejecución de propuesta, teniendo implicancia en el logro del objetivo (finalización del proyecto), lo que se vincula directamente con la satisfacción registrada por los participantes en la encuesta.

2. Valoración del desplazamiento espacial, que supuso la posibilidad de transitar de un lugar a otro durante el desarrollo de la actividad

3. La dinámica de funcionamiento del taller fue diferente en estudiantes y profesores, lo que se vincula con el rol social asumido por cada grupo. Si bien los estudiantes valoraron el hecho de haber entablado una relación horizontal con el profesor guía, la participación fue más alta por parte de los docentes, quienes, desde el inicio de la actividad, se hicieron parte del proceso enseñanza aprendizaje aportando conocimientos y reflexiones desde sus diferentes áreas disciplinares.

4. Las bio-herramientas utilizadas en el espacio exterior (juego, técnicas de respiración, técnicas de enraizamiento) generaron un clima positivo y de excelente predisposición en los participantes de ambos talleres, lo que se tradujo en una alta motivación para desarrollar las actividades en el espacio interior (taller de arquitectura). Especialmente entre los docentes, se manifestó una predisposición al juego (lúdica), en la identidad y en la emocionalidad.

5. La didáctica de taller proyectual favoreció el trabajo colaborativo entre los participantes, posibilitando la comunicación y el intercambio de roles y funciones entre pares. También se observó un efecto reflexivo en las partes involucradas. 
A modo de conclusión, se puede señalar el importante aporte que supone el trabajo con nuevas metodologías aplicables en el aula. En particular, se considera que la metodología de didáctica de proyecto puede convertirse en una herramienta transferible, que efectivamente facilite el aprendizaje de manera más integral y holística, en tanto supone la integración de los diferentes planos que componen al hombre y una visión no fragmentada del conocimiento. El modelo ensayado en esta investigación, para el logro efectivo de los objetivos propuestos, requiere considerar varios aspectos:
- Una organización rigurosa de la propuesta de trabajo.

- El conocimiento acabado de los diferentes ámbitos del saber, que se articulan durante el ejercicio de proyecto.

- La disposición del docente a empatizar amable y cordialmente con las personas con quienes trabaja.

- Mantener una relación de horizontalidad.

- Generar un clima de trabajo tranquilo, cómodo y cálido.

- Innovar creativamente con nuevas herramientas y áreas de conocimiento, que se integren con el propósito de una formación relacional.
En último lugar, se observa que el modelo didáctico proyectual puede concebirse como una herramienta útil, especialmente para los docentes que requieran de nuevas metodologías de enseñanza, dando respuesta de ese modo a las exigentes trasformaciones que no solo promueve el sistema nacional de educación, sino también los propios estudiantes, quienes deben adaptarse a un mundo cada vez más dinámico y complejo.

\section{REFERENCIAS}

Barberousse P. 2008 "Fundamentos teóricos del pensamiento complejo de Edgar Morin" Revista electrónica Educare, 12 (2): 95-113

Barfield, T., 2001. Diccionario de antropologia. Barcelona: Edicions Bellaterra

Bengoa, José. 1992. El testigo. Apuntes de clase de un Curso de Historias de Vida. Santiago de Chile: Ediciones Sur

Bourdieu, P., 2008. Una invitación a la sociología reflexiva. Buenos Aires: Siglo XXI Editores.

Brovetto, J., 1999. "La educación superior en Iberoamérica: Crisis, debates, realidades y transformaciones en la última década del siglo XX." Revista iberoamericana de educación. 21, 41-53.

Dewey, J., 1965. La Ciencia y la Educación. Buenos Aires: Losada.

Eisner, E., 1994. Cognition and curriculum reconsidered. Nueva York: Teachers College Press.

Galdames, R. Moffat, S. y Cisternas, E, 2014 "La construcción participativa del Observatorio Prospectivo de

Educación y Desarrollo Regional del Biobío." Estudios Regionales, 36.

Geertz, C., 1987. La interpretación de las culturas. DF México: Gedisa.

Hammersley, M. y Atkinson, P. 1994. Etnografía. Métodos de Investigación. Barcelona: Paidós.

Lagos, R., 2016، "Armónicos: Sólidos platónicos como base de modelo didáctico de iniciación al proyecto en

Arquitectura." Arquitetura Revista, 12 (1): $48-57$.

Lagos, R., 2013. "Enseñanza de la arquitectura, movimiento universitario y reformas en Chile (1964-1973).

Revista 180, 32

Lagos, R., 2012. "Prefacio de Maestría del proyecto, de Jean Francois Mabardi." Concepción: Ediciones de la

Universidad del Bio-Bio.
Mauss. M. 2006 Manual de etnografía. Buenos Aires: Fondo de Cultura Económica.

Mac Lean, P., 1990. The triune brain in evolution: role in paleocerebral functions. Nueva York: Plenum Press Morin. E., 1999. Los siete saberes necesarios para la educación del futuro. Paris: UNESCO.

Morin, E., 1994. Introducción al pensamiento complejo. España: Gedisa.

Paymal, N., 2012. ¿Qué es el desarrollo integral del Ser en la educación? Pedagogía 3000. La Paz: Editorial Ox La- Hun.

Paymal, N., 2010. Guía práctica para docentes, padres y uno mismo. Pedagogía 3000. La Paz: Editorial Ox La-Hun.

Rojas, P., 2017. “Una Educación Artística para Desarrollar el Bienestar Subjetivo. La Experiencia Chilena." Revista Internacional de Educación para la Justicia Social (RIEJS), 6 (1): 199-216.

Román, M., 2003. "Factores culturales que determinan el aprendizaje y rendimiento de los niños y niñas de las escuelas subvencionadas por el Estado." Simposio Antropología y Educación. Tomo ll, Actas del $4^{\circ}$ Congreso Chileno de Antropología. Los desafíos de la Antropología. Globalización, Sociedad Moderna y Diferencia. Santiago de Chile: LOM Ediciones, 955-963.

Santos, M. 2000. "El pensamiento complejo y la pedagogía. Bases para una teoria holistica de la educación" Estudios pedagógicos, 26, 133-148

Schön, D. 1992. La formación de profesionales reflexivos. Hacia un nuevo diseño de la enseñanza y el

aprendizaje en las profesiones. Barcelona: Paidós. 